\title{
A Secure Message Transmission Scheme in an Extended Network of Crossed Cubes
}

\author{
Bao-lei CHENG ${ }^{1,2, a}$, Ting PAN ${ }^{1, b}$, Jian-xi $\mathrm{FAN}^{1, \mathrm{c}}$, Jing-ya ZHOU ${ }^{1, \mathrm{~d}}$ \\ and Zhao LIU ${ }^{1, e}$ \\ ${ }^{1}$ School of Computer Science and Technology, Soochow University, Suzhou 215006, \\ China \\ ${ }^{2}$ Provincial Key Laboratory for Computer Information Processing Technology, \\ Soochow University, China

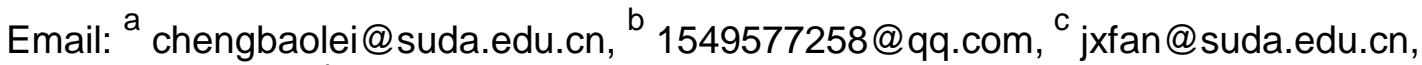 \\ djy_zhou@suda.edu.cn, ${ }^{\mathrm{e}}$ liuzhao@suda.edu.cn
}

Keywords: Extended network of crossed cubes, Secure message transmission scheme, Independent spanning trees, Vertex-disjoint paths.

\begin{abstract}
Secure message transmission schemes in interconnection networks can be achieved by constructing multiple vertex-disjoint paths or independent spanning trees. The extended network of crossed cube (ECC for short) has the advantages such as easy to be deployed and low ratio between number of vertices and diameter. In this paper, we have studied a secure message transmission scheme in ECC by constructing $n$ independent spanning trees in $S_{n}$ and proposed a constructive algorithm with the time complexity $O(N)$, where $N$ is the number of $S_{n}$. Furthermore, the maximum length of the vertex-disjoint paths between any two vertices is shown to be $2 n+3$. In addition, simulation of vertex-disjoint paths based on JUNG has also been discussed.
\end{abstract}

\section{Introduction}

Multiple vertex-disjoint paths between arbitrary two vertices in interconnection networks can be used to transmit different parts of a message. Every part of the message can use its own encryption methods in its corresponding path with safety. Such vertex-disjoint paths can be constructed based on the multiple independent spanning trees (ISTs) [1,2]. Towards the conjecture that for any $n$-connected graph $G(n \geq 1)$, there are $n$ ISTs rooted at an arbitrary vertex on $G[1,2]$, it was only solved for $n \leq 4[1,2,3,4]$, but remains open for $n \geq 5$. Thus, the results on special graphs are still the focus of researchers and many results have been obtained, such as hypercubes $[5,6]$, crossed cubes [7], even networks [8], odd networks [9], folded hyper-stars [10], multidimensional torus networks [11], recursive circulant graphs [12], Gaussian networks [13], 2-chordal rings [14], and so on.

In comparison with the hypercube, the crossed cube has good properties such as lower vertex degree and diameter, higher connectivity, symmetry, and etc $[15,16]$. But in the real world, it is difficult to build interconnection networks based on crossed cubes, because according to the structure of crossed cubes, a computer is needed to connect many neighbor computers. However, to install two Ethernet cards in a computer is feasible and as a consequence the extended crossed cube ECC is proposed [17], which is easy to be deployed and has low ratio between number of vertices and diameter.

In this paper, we study a secure message transmission scheme in the ECC by 
constructing multiple ISTs rooted at an arbitrary vertex. Firstly, algorithms to construct spanning trees and ISTs in $S_{n}$ are proposed. Then, $n$ vertex-disjoint paths between any two vertices are discussed, the maximum length of which is shown to be no more than $2 n+3$. Finally, we have simulated the vertex-disjoint paths in $S_{4}$ based on JUNG.

\section{Preliminaries}

A binary string $v$ of length $n$ will be written as $v_{n-1} v_{n-2} \ldots v_{0}$, where $v_{n-1}$ and $v_{0}$ are the most significant bit and the least significant bit, respectively. The bit $v_{i} \in\{0,1\}$ is called the $i$ th bit of $v$ for integer $i$ with $0 \leq i \leq n-1$. The $n$-dimensional crossed cube, denoted as $C Q_{n}$, has $2^{n}$ vertices. Each vertex in $C Q_{n}$ is represented by a unique binary string of length $n$. We adopt the extended network of crossed cube ECC, denoted as $S_{n}$, from [17].

Definition 2.1. [17] $S_{n}$ is defined as follows. $S_{1}$ is a complete graph on two vertices $0[0], 1[0] . S_{2}$ is a cycle with the vertices set $\{00[0], 00[1], 01[0], 01[1], 10[0], 10[1]$, $11[0], 11[1]\}$ and edge set $\{(00[0], 00[1]),(00[1], 10[1]),(10[0], 10[1]),(10[0], 11[0])$, (11[0], 11[1]), (11[1], 01[1]), (01[0], 01[1]), (00[0], 01[0])\}. For $n \geq 2, S_{n}$ is the network to extend every vertex in $C Q_{n}$ into a complete graph on $n$ vertices. The vertex set of $S_{n}$ is denoted as $V\left(S_{n}\right)=\left\{u[i] \mid u \in V\left(C Q_{n}\right)\right.$, for $\left.i=0,1, \ldots, n-1\right\}$. For any two vertices $u[j] \in V\left(S_{n}\right)$ and $v[k] \in V\left(S_{n}\right)$, where $0 \leq k, j \leq n-1,(u[j], v[k]) \in V\left(S_{n}\right)$ if and only if the following conditions hold:

(1) $j \neq k$ and $u=v$, and

(2) $j=k$ and $(u, v) \in E\left(C Q_{n}\right)$.

Let $V(G), E(G), \delta(G), \lambda(G)$, and $D(G)$ denote the vertex set, edge set, the minimum degree, the edge-connectivity, and the diameter, respectively. As a $n$-regular graph, $S_{n}$ has the following properties.

Lemma 2.1. [17] $S_{n}$ has $n * 2^{n}$ vertices and $n^{2} * 2^{n-1}$ edges.

Lemma 2.2. [17] $\delta\left(S_{n}\right)=n$ for $n \geq 1$.

Lemma 2.3. [17] $\lambda\left(S_{n}\right)=n$ for $n \geq 1$.

Lemma 2.4. [17] $D\left(S_{n}\right) \leq n+3$.

\section{Independent Spanning Trees}

\section{Broadcasting Tree in $S_{n}$}

When we want to distribute a message from a vertex to all other vertices in $S_{n}$, broadcasting tree can be used to distribute the message in $S_{n}$. Now we give an algorithm to construct a spanning trees $T$. Let $u(k)$ be the root vertex, where $0 \leq k \leq n-1$ and $u \in V\left(C Q_{n}\right)$. Clearly, $u$ can be written as $u_{n-1} u_{n-2} \ldots u_{0}$. We use $N_{k}(u[k])$ to denote the $k$-neighbor of $u[k]$.

Algorithm SpanningTree

Input: $u[k]$ be the root vertex, where $0 \leq k \leq n-1$ and $u \in V\left(C Q_{n}\right)$; 
Output: A tree $T$ rooted at $u[k]$ on $S_{n}$;

Begin

Step 1:

1: Let $V(T)=\left\{v[m] \mid\right.$ for all $v \in V\left(C Q_{n}\right)$ and all $\left.0 \leq m \leq n-1\right\}$.

2: Connect $u[i]$ to $u[k]$ for all $0 \leq i \leq k-1$ and $k+1 \leq i \leq n-1$.

\section{Step 2:}

3: for $j=0$ to $n-1$ do

4: $\quad$ for each vertex $v[j] \in V(T)$ do

5: $\quad E(T)=E(T) \cup\left(v[j], N_{j}(v[j])\right)$.

6: $\quad$ Let $m[j]=N_{j}(v[j])$.

7: $\quad$ Connect $m[i]=m[j]$ for all $0 \leq i \leq j-1$ and $j+1 \leq i \leq n-1$.

8: $\quad$ end for

\section{9: end for}

\section{End}

Figure 1 shows the construction procedures of a spanning tree rooted at 100[2] by Algorithm SpanningTree in $S_{3}$. Firstly, by Step 1, the vertices in $T$ is created. The edges in the set $\{(100[2], 100[0]),(100[2], 100[1])\}$ are also constructed (See Figure 1(a)). Secondly, Figure 1(b), Figure 1(c), and Figure 1(d) show the three iterations of construction of edges. The edge sets are constructed as \{(100[0], 101[0]), (101[0], 101[2]), (101[0], 101[1])\},\{(101[1], 111[1]), (111[1], 111[2]), (111[1], 111[0]), (100[1], 110[1]), (110[1], 110[2]), (110[1], 110[0])\}, \{(100[2], 000[2]), (000[2], $000[1]),(000[2], 000[0]),(101[2], 011[2]),(011[2], 011[1]),(011[2], 011[0]),(111[2]$, 001[2]), (001[2], 001[1]), (001[2], 001[0]), (110[2], 010[2]), (010[2], 010[1]), (010[2], $010[0])\}$, respectively.

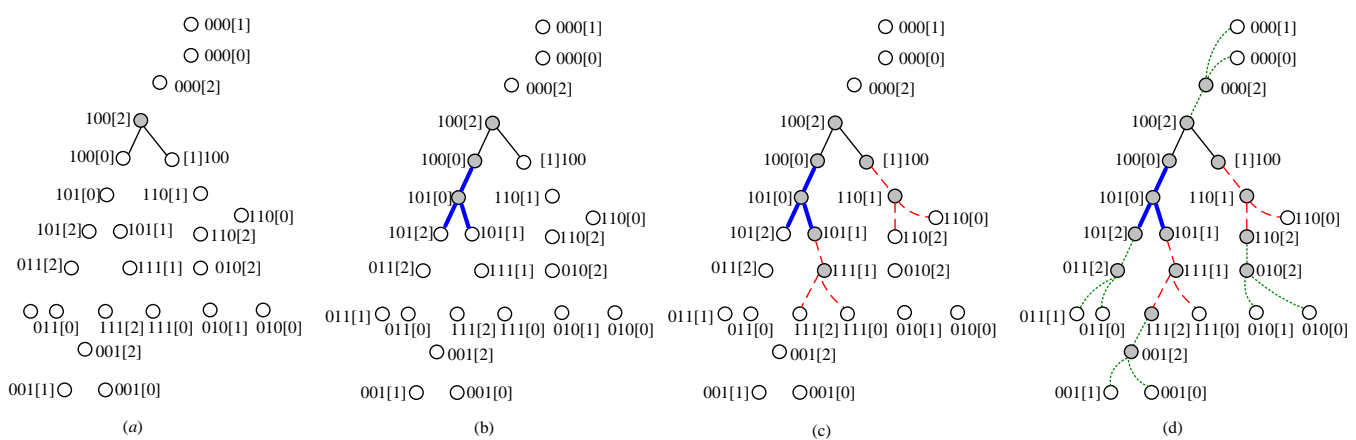

Figure 1. Demonstration of different steps of edges constructed by Algorithm SpanningTree in $S_{3}$ : (a) Step 1; (b) First iteration Step 2;

(c) Second iteration of Step 2; (d) Third iteration of Step 2.

Here, we also use line types such as solid line, bold solid line, dashed lines, dotted lines to denote the above edges in the four sets, respectively. By Algorithm SpanningTree, $V(T)=\left\{v[m] \mid\right.$ for all $v \in V\left(C Q_{n}\right)$ and all $\left.0 \leq m \leq n-1\right\}$. Based on Definition 2.1, it is clear that $T$ contains all vertices in $S_{n}$, which is extended from $C Q_{n}$ from the aspect of vertices, thus it also has potential recursive construction. Using the recursive relation, we can verify that $T$ is an undirected graph in which any two 
vertices are connected by exactly one path. Thus, the following lemma holds. By computing the relation of edges and iteration times, the height of $T$ is $2 n+1$. Then, the following theorem holds.

Theorem 3.1. Given the input $u[k]$ as the root, where $0 \leq k \leq n-1$ and $u \in V\left(C Q_{n}\right)$, $T$ constructed by Algorithm SpanningTree is a spanning tree on $S_{n}$ with the height $2 n+1$.

In Algorithm SpanningTree, line 4 " for each vertex $v[j] \in V(T)$ do " can be parallized, thus the broadcasting tree can be constructed by $n$ steps with the time complexity $O(n)$. The unicast path between any two vertices and the multicast tree rooted at an arbitrary vertex can also be obtained. By Algorithm SpanningTree, the length of the unicast path and the height of the multicast tree are no more than $2 n+1$.

Independent Spanning Trees in $S_{n}$

In the above section, the root vertex of the spanning trees $T$ constructed by Algorithm SpanningTree has multiple children. But according to the definition of independent spanning trees (ISTs) [1,2], if we want to obtain $n$ ISTs in $S_{n}$, then the root of every IST can have only one child.

Now we give a revised algorithm to construct independent spanning trees in $S_{n}$ based on Algorithm SpanningTree.

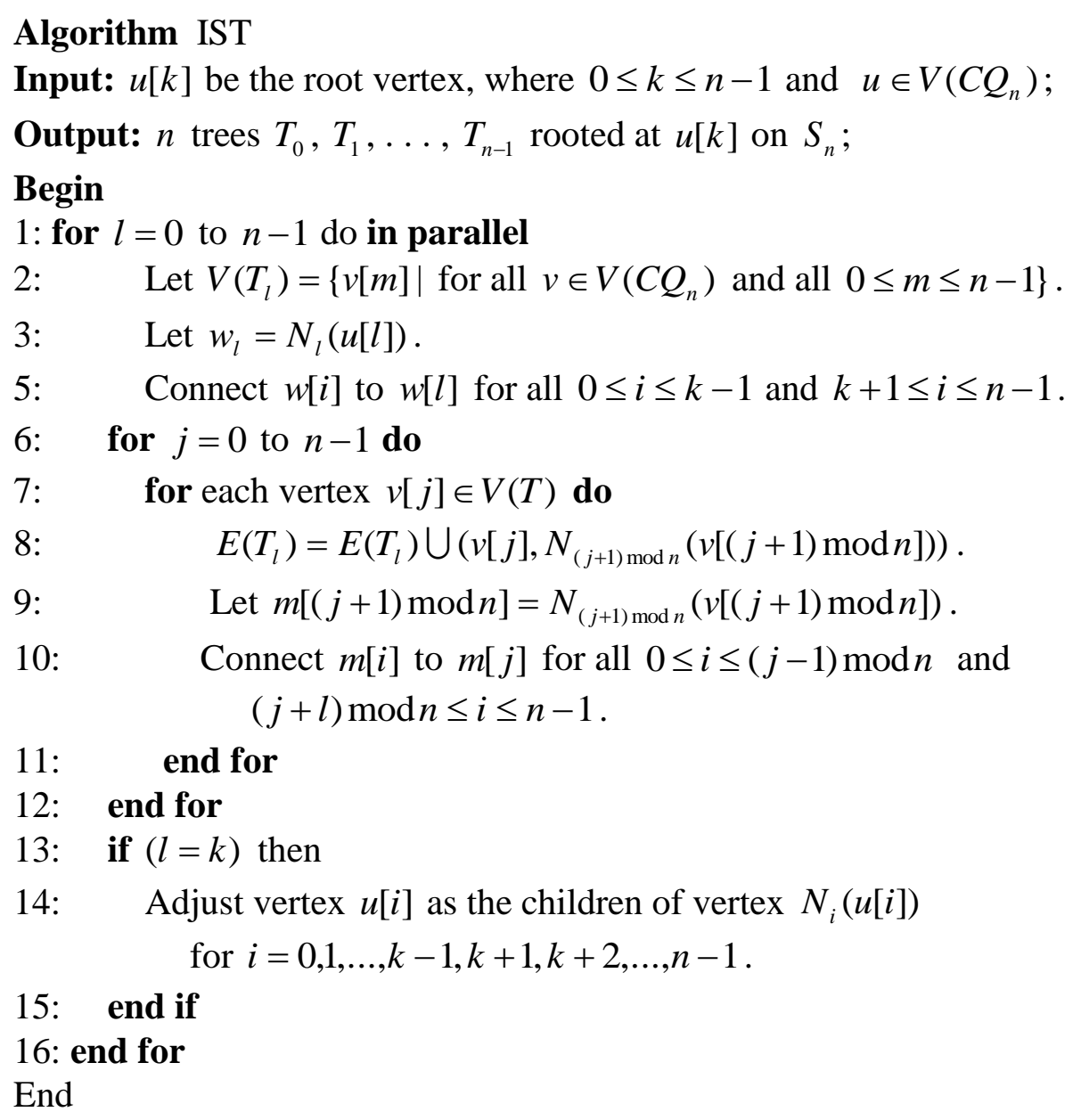


Figure 2 shows the three ISTs rooted at 000[1] in $S_{3}$ constructed by Algorithm IST. The construction of $T_{0}$ and $T_{2}$ is similar to the tree constructed by Algorithm SpanningTree(See Figure 2(a) and Figure 2(c)). Every tree can be constructed by several iterations. For tree $T_{1}$, based on lines 13-15 of Algorithm SpanningTree, we have deleted the edges $(000[1], 000[0])$ and $(000[1], 000[2])$. Two new edges $(100[2]$, $000[2])$ and (001[0], 000[0]) are appended(See Figure 2(b)). Since the $n$ trees of Algorithm IST can be constructed in parallel, based on Definition 2.1 and the ISTs results in $C Q_{n}$, we can prove that the following theorem holds.

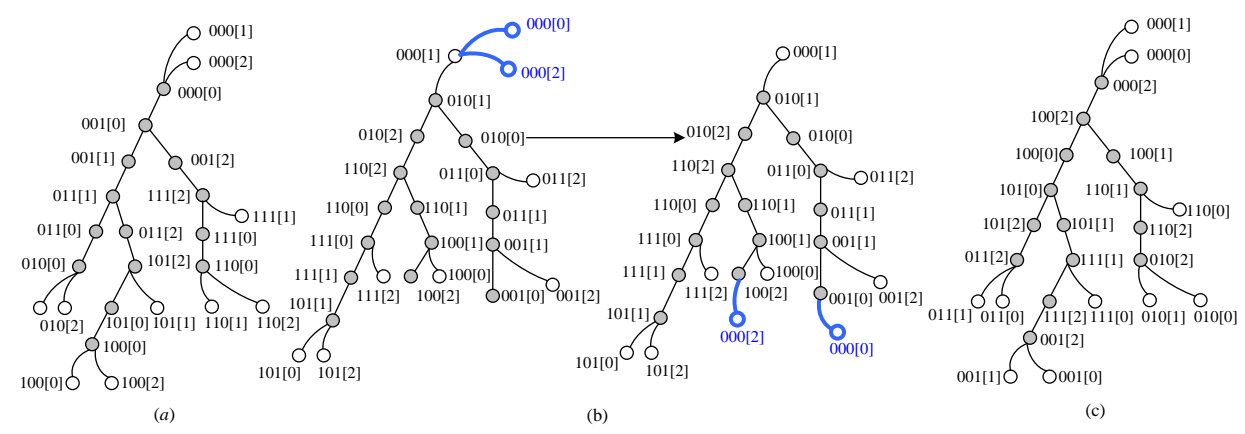

Figure 2. Three ISTs rooted at $000[1]$ in $S_{3}$ :(a) $T_{0}$; (b) $T_{1}$; (c) $T_{2}$

Lemma 3.1. Algorithm IST constructs $n$ trees rooted at vertex $u[k]$ in $O(N)$ time in $S_{n}$, where $N=n * 2^{n}$ is the vertex number of $S_{n}$.

Theorem 3.2. For an integer $n$ with $n \geq 1$ and an arbitrary vertex $u[k]$ on $S_{n}, T_{0}$, $T_{1}, \ldots$, and $T_{n-1}$ constructed by Algorithm IST are $n$ ISTs rooted at vertex $u[k]$ in $S_{n}$.

\section{Discussion of Vertex-disjoint Paths in $S_{n}$}

Based on Algorithm IST, any vertex can be the root vertex of ISTs is arbitrary, thus we can construct vertex-disjoint paths between any two vertices. The three vertex-disjoint paths between vertices $000[1]$ and 101[1] are as follows.

$$
\begin{aligned}
& 000[1] \rightarrow 000[0] \rightarrow 001[0] \rightarrow 001[1] \rightarrow 011[1] \rightarrow 011[2] \rightarrow 101[2] \rightarrow 101[1] \\
& 000[1] \rightarrow 010[1] \rightarrow 010[2] \rightarrow 110[2] \rightarrow 110[0] \rightarrow 111[0] \rightarrow 111[1] \rightarrow 101[1] \\
& 000[1] \rightarrow 000[2] \rightarrow 100[2] \rightarrow 100[0] \rightarrow 101[0] \rightarrow 101[1]
\end{aligned}
$$

The iterative sequence in Algorithm IST is $0,1, \ldots, n-1$. Since the root vertex of Algorithm IST is arbitrary, if we change the sequence, we can obtain another set of three vertex-disjoint paths between any two vertices. For example, using the sequence 0 , 2, 1, we can obtain another three vertex-disjoint paths between 000[1] and 110[0].

$$
\begin{aligned}
& 000[1] \rightarrow 000[0] \rightarrow 001[0] \rightarrow 001[2] \rightarrow 111[2] \rightarrow 111[1] \rightarrow 101[1] \\
& 000[1] \rightarrow 010[1] \rightarrow 010[0] \rightarrow 011[0] \rightarrow 011[2] \rightarrow 101[2] \rightarrow 101[1] \\
& 000[1] \rightarrow 000[2] \rightarrow 100[2] \rightarrow 100[1] \rightarrow 101[1]
\end{aligned}
$$

Observing the above two set of vertex-disjoint paths, the lengths of the former are 7, 7 , and 5, respectively. The total length of the paths is 19 . The lengths of the latter are 6 , 6 , and 4, respectively, and the total length of which is 16 , which is lower than the former. The maximum lengths of the paths in two sets are 7 and 6, respectively, both of which are lower than the height of any of the IST. 
Now we discuss the maximum length of the vertex-disjoint paths. By Definition 2.1, for any two vertices $v[i]$ and $v[j]$ in $S_{n}$, where $0 \leq i<j \leq n-1, v[i]$ is connected with $v[j]$. By Algorithm IST, the number of iterations is $n$, the root vertex is the child of the input vertex. Thus, for any tree, the number of the vertices in the path from the root vertex to the farthest leaf vertex is $2 *(n+2)-1=2 n+3$. Thus, we have the following theorem.

Theorem 3.3. For any two vertices $u[k]$ and $v[i](v[i] \neq u[k])$ in $S_{n}$, the maximum length of the $n$ vertex-disjoint paths between the two vertices is $2 n+3$.

\section{Simulation of Vertex-disjoint Paths}

Now we simulate the construction of vertex-disjoint paths between two vertices based on Algorithm IST and Java Universal Network/Graph Framework (JUNG).

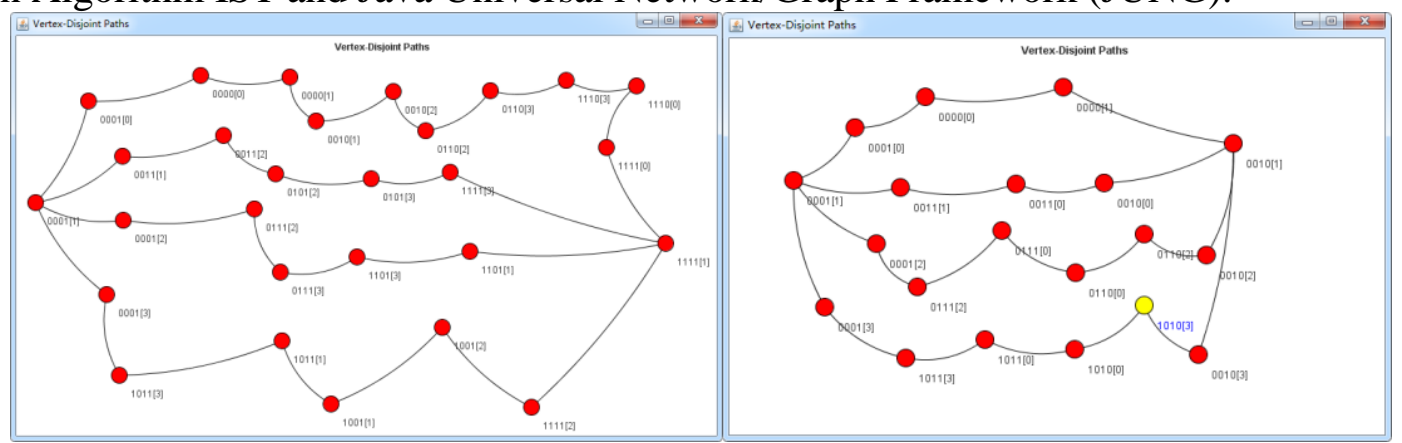

(a)

(b)

Figure 3. Vertex-disjoint paths between vertices (a) 0000[1] and 1111[1]; (b) 0000[1] and 0010[1]

Figure 3(a) shows the four vertex-disjoint paths between vertices 0001[1] and $1111[1]$. We can see that the maximum length of the four paths is 11 and the total length of the four paths is 30 . In another case, let 0010 [1] be the destination vertex, the maximum length of the four paths becomes 7 and the total length of the four paths is 22 , which is shown in Figure 3(b).

Here, if we transmit secure data in the latter four paths, the efficiency is higher than the former four paths. In addition, in a large network of computers based on $S_{n}$. If we want to choose different computers to backup data, the maximal length of the path is lower, then the efficiency may be higher. Thus, we can choose the proper vertices based on our real needs.

\section{Conclusions}

In this paper, we have discussed a secure message distribution scheme in an extended network of crossed cubes ECC by constructing independent spanning trees. Based on the set of independent spanning trees constructed by Algorithm IST, we can also obtain the $n$ vertex-disjoint paths between any two vertices, the maximum length of which is $2 n+3$. 


\section{Acknowledgment}

This work is supported by National Natural Science Foundation of China (No. 61572337 and No. 61502328), China Postdoctoral Science Foundation Funded Project (No. 2015M581858), the Natural Science Foundation of the Jiangsu Higher Education Institutions of China (No. 14KJB520034 and No. 15KJB520032), and the Jiangsu Planned Projects for Postdoctoral Research Funds (No. 1501089B) and the eighteenth batch of college students extracurricular academic research fund projects.

\section{References}

[1] A. Itai and M. Rodeh, The multi-tree approach to reliability in distributed networks, Inform. Comput. 79(1) (1988) 43-59.

[2] A. Zehavi and A. Itai, Three tree-paths, J. Graph Theor. 13(2) (1989) 175-188.

[3] J. Cheriyan and S. N. Maheshwari, Finding nonseparating induced cycles and independent spanning trees in 3-connected graphs, J. Algorithm. 9(4) (1988) 507-537.

[4] S. Curran, O. Lee, and X. Yu, Finding four independent trees, SIAM J. Comput. 35(5) (2006) 1023-1058.

[5] K. Obokata, Y. Iwasaki, F. Bao, and Y. Igarashi, Independent spanning trees of product graphs and their construction, IEICE T. Fund. Electr. E79-A(11)(1996) 1894-1903.

[6] S.-M. Tang, Y.-L. Wang, and Y.-H. Leu, Optimal independent spanning trees on hypercubes, J. Inf. Sci. Eng. 20(1) (2004) 143-155.

[7] B. Cheng, J. Fan, X. Jia, and J. Wang, Dimension-adjacent trees and parallel construction of independent spanning trees on crossed cubes, J. Parallel Distr. Com. 73(5) (2013) 641-652.

[8] J.-S. Kim, H.-O. Lee, E. Cheng, and L. Lipták, Independent spanning trees on even networks, Inf. Sci. 181(13) 2892-2905.

[9] J.-S. Kim, H.-O. Lee, E. Cheng, and L. Lipták, Optimal independent spanning trees on odd graphs, J. Supercomput. 56(2) (2011) 212-225.

[10] J.-S. Yang and J.-M. Chang, Independent spanning trees on folded hyper-stars, Networks 56(4) (2010) 44-53.

[11] S.-M. Tang, J.-S. Yang, Y.-L. Wang, and J.-M. Chang, Independent spanning trees on multidimensional torus networks, IEEE Trans. Comput. 59(1)(2010) 93-102.

[12] J.-S. Yang, J.-M. Chang, S.-M. Tang, and Y.-L. Wang, On the independent spanning trees of recursive circulant graphs $\mathrm{G}\left(\mathrm{cd}^{\mathrm{m}}, \mathrm{d}\right)$ with $\mathrm{d}>2$," Theoret. Comput. Sci. 410 (21-23) (2009) 2001-2010.

[13] Z. Hussain, B. AlBdaiwi, and A. Cerny, Node-independent spanning trees in Gaussian networks, Proceedings of the International Conference on Parallel and Distributed Processing Techniques and Applications (PDPTA). The Steering Committee of The World Congress in Computer Science, Computer Engineering and Applied Computing (WorldComp), 2016, 24-29.

[14] Y. Hamada, Independent spanning trees of 2-chordal rings, IEICE T. Fund. Electr. 99(1) (2016) 355-362. 
[15] C.-P. Chang, T.-Y. Sung, and L.-H. Hsu, Edge congestion and topological properties of crossed cubes, IEEE T. Parall. Distr. 11(1) (2000) 64-80.

[16] K. Efe, A variation on the hypercube with lower diameter, IEEE T. Comput. 40(11) (1991) 1312-1316.

[17] B.-L. Cheng, J.-X. Fan, J.-W. Yang, Z. Liu, and J.-Y. Zhou, An extended network of crossed cubes, Proc. 2016 International Conference on Computer Science and Information Security (CSIS 2016), 590-596 April 16-17, 2016, Nanjing, China. 